\title{
Diagnostic value of KL-6 in idiopathic interstitial pneumonia
}

\author{
Peiyan Zheng", Xiaoqing Liu", Huimin Huang, Zijun Guo, Ge Wu, Haisheng Hu, Chuanxu Cai, Wenting \\ Luo, Nili Wei, Qian Han, Baoqing Sun
}

Department of Allergy and Clinical Immunology, Guangzhou Institute of Respiratory Health, State Key Laboratory of Respiratory Disease, National Clinical Research Center for Respiratory Disease, The First Affiliated Hospital of Guangzhou Medical University, Guangzhou 510120, China

Contributions: (I) Conception and design: P Zheng, B Sun; (II) Administrative support: G Wu, H Hu, C Cai, W Luo; (III) Provision of study materials or patients: X Liu, H Huang, Z Guo; (IV) Collection and assembly of data: P Zheng, Q Han, N Wei; (V) Data analysis and interpretation: P Zheng, X Liu, Q Han, B Sun; (VI) Manuscript writing: All authors; (VII) Final approval of manuscript: All authors.

\#These authors contributed equally to this work.

Correspondence to: Baoqing Sun; Qian Han. State Key Laboratory of Respiratory Disease, the First Affiliated Hospital of Guangzhou Medical University, 151 Yanjiangxi Road, Guangzhou 510120, China. Email: sunbaoqing@vip.163.com; hanqian1020@yahoo.com.

Background: Idiopathic interstitial pneumonia (IIP) can induce type II alveolar epithelial cell proliferation and pulmonary basement membrane damage and subsequent release of Krebs von den Lungen-6 antigen (KL-6) to the bloodstream. This study investigated the diagnostic and prognostic value of serum KL-6 levels for IIP.

Methods: One hundred five patients with lung disease were divided into IIP ( $\mathrm{n}=75)$ and non-IIP groups $(\mathrm{n}=30)$ according to pathological and computed tomography findings. Serum KL-6 levels were evaluated in blood samples from all subjects. Nineteen IIP group patients were also subjected to a longitudinal study of disease progression and serum KL-6 levels over time.

Results: Serum KL-6 levels were significantly higher in the IIP group vs. the non-IIP group [1,096.0 (565.0-1,544.0) vs. 226.0 (173.5-346.5) U/mL; P<0.01]. Within the IIP group, serum KL-6 levels differed significantly between patients with and without concomitant disease or pulmonary infection $(Z=-2.475$, $\mathrm{P}=0.013$ ). In a receiver operating characteristic (ROC) curve analysis, the area below the curve for serum KL-6 was 0.911 [95\% confidence interval (CI): 84.7-97.5\%, $\mathrm{P}<0.001$ ], indicating a good diagnostic performance for IIP, with a cut-off level of $485 \mathrm{U} / \mathrm{mL}$, sensitivity of $85.33 \%$, specificity of $90.00 \%$, positive predictive value (PPV) of 95.52\%, negative predictive value (NPV) of $71.05 \%$, and Kappa value of 0.70 . Accordingly, the serum KL-6 and clinical diagnostic results were consistent. Moreover, in the longitudinal study, the serum KL-6 levels differed significantly from before to after treatment in patients with exacerbated or improved disease $(\mathrm{P}=0.004$ and $\mathrm{P}=0.043$, respectively), whereas no obvious changes were observed in patients with stable disease $(\mathrm{P}=0.692)$.

Conclusions: The serum KL-6 level is a valuable and significant diagnostic marker of IIP and a useful predictor of clinical prognosis.

Keywords: Serum KL-6; diagnostic test; idiopathic interstitial pneumonia (IIP)

Submitted Sep 27, 2017. Accepted for publication Jul 11, 2018.

doi: $10.21037 /$ jtd.2018.07.54

View this article at: http://dx.doi.org/10.21037/jtd.2018.07.54

\section{Introduction}

Interstitial lung diseases (ILDs) are diffuse parenchymal lung diseases that affect the lung interstitium, pulmonary alveoli, and bronchioles. ILDs include idiopathic interstitial pneumonia (IIP), collagen vascular disease-interstitial pneumonia (CVD-IP), and hypersensitive pneumonia (HP). Of these, IIP, a refractory disease with a poor prognosis, 
is the most common form of ILD. According to the 2013 American Thoracic Society (ATS)/European Respiratory Society (ERS) joint multidisciplinary classification of IIPs, common forms include idiopathic pulmonary fibrosis (IPF) and idiopathic nonspecific interstitial pneumonia (INSIP), as well as others such as respiratory bronchiolitis-interstitial lung disease (RB-ILD), desquamative interstitial pneumonia (DIP), cryptogenic organising pneumonia (COP), and acute interstitial pneumonia (AIP) (1).

Currently, definitive epidemiological data regarding IIPs remain unavailable. In the USA, a study conducted in the state of New Mexico (2) reported that the incidence of IIP is low among children but increases with age, with prevalence rates of $6-14.6 / 100,000$ and $175 / 100,000$ in the overall population and among those aged $>75$ years, respectively. Similarly, little is known about the prevalence of IPF, a common form that accounts for $52.6 \%$ of IIP cases. Studies have reported IPF prevalence rates of $10.5 / 100,000$ and $14.0-42.7 / 100,000$ in Japan and the USA, respectively (3), and although national statistical data remain unavailable in China, estimates indicate that the Chinese population includes $>300,000$ patients with IPF. Currently, there is no effective treatment for IPF, and the median post-diagnostic survival duration is approximately $2-3$ years. Acute exacerbation of IPF (AE-IPF) is the major cause of IPFrelated mortality, accounting for $85 \%$ of deaths (3-5).

Presently, a definitive diagnosis of IIP is based on clinical-radiologic-pathologic (CRP) factors (6). Lung biopsy, the gold-standard diagnostic method, tends to be performed only after the appearance of clinical signs, causes injury to patients, and is subject to regional differences. Accordingly, biopsy remains unpopular (7). Imaging examinations, such as high-resolution computed tomography (HRCT), expose patients to radiation and are therefore unsuitable for repeated examinations conducted to perform dynamic monitoring at short intervals. Pulmonary function tests (PFTs), which are affected by multiple factors and require a high degree of patient cooperation, are also unsuitable for the routine testing of critically ill patients. None of the above methods yield a significant level of early diagnostic value. However, the early administration of immunosuppressive and anti-fibrotic therapies can prevent and delay the progression of pulmonary alveolitis to irreversible end-stage fibrosis. Therefore, a simple, patientfriendly diagnostic and prognostic marker of IIP onset, progression, and severity is needed. Ideally, this marker would non-invasively facilitate the clinical diagnosis and dynamic monitoring of IIPs.

In 1985, Kohno identified the Krebs von den Lungen-6 (KL-6) antigen, a high-molecular-weight glycoprotein (molecular weight: $>1$ million $\mathrm{kDa}$ ), in a Japanese study of pulmonary adenocarcinoma (7). KL-6 was initially studied as a tumour marker but was later found to exert chemotactic and anti-apoptotic effects on fibroblast cells (8). Notably, interstitial pneumonia can induce the proliferation of type II alveolar epithelial cells, causing pulmonary basement membrane damage and increased vascular permeability that enhance the release of KL-6 to the bloodstream (9). KL-6 expression is not limited to type II alveolar epithelial, bronchial glandular epithelial, and bronchial serous gland cells but has also been detected in other normal cell types (e.g., pancreatic and breast ducts). A previous study also observed high levels of KL-6 in the regenerated type II alveolar epithelial cells and sera of ILD patients $(10,11)$. However, few previous studies have compared the serum KL-6 levels of patients with IIP and other pulmonary diseases (e.g., pulmonary sarcoidosis, respiratory tract infections, bronchitis, etc.) or evaluated the relationships of changes in serum KL-6 levels with the clinical outcomes of IIPs. This study aimed to explore the clinical significance and potential use of KL-6 as a biomarker for the diagnosis and monitoring of IIP.

\section{Methods}

\section{Research participants}

One hundred five patients diagnosed with lung disease in the Department of Respiration of the First Affiliated Hospital of Guangzhou Medical University from April 2014 to March 2016 were recruited. The recruits comprised 75 patients with IIP (IIP group), including 20 with IPF, 13 with NSIP, and 28 with others diseases (including RB-ILD, DIP, COP, and AIP). These patient subgroups did not exhibit significant differences in KL-6 levels, as shown in Figure S1. The control group (non-IIP group) comprised 30 patients diagnosed with other pulmonary diseases during the same time period, including 7 with bronchitis; 5 with pulmonary infection; 4 each with pneumonia, pulmonary sarcoidosis, or respiratory tract infection; and 3 each with chronic obstructive pulmonary disease or pneumothorax. This study was approved by the Medical Ethics Committee of The First Affiliated Hospital of Guangzhou Medical University (ethics approval no. gyfyy-2016-73). All experiments were performed in accordance with the relevant guidelines and regulations of the Ethics Committee of First Affiliated Hospital of Guangzhou Medical University. 


\section{Study inclusion criteria}

The study subjects (including the non-IIP group) comprised male and female patients older than 18 years who had been diagnosed via HRCT and histopathological evaluations. The inclusion criteria were defined in accordance with the 2013 ATS/ERS International Multidisciplinary Consensus Classification of IIPs (ATS/ERS Classification of IIPs) (1).

\section{Study exclusion criteria (12)}

Patients with other types of diffuse ILDs (e.g., sarcoidosis, HP, and connective tissue disease-associated ILD) were excluded from the study. Patients with pulmonary diseases who met the following criteria were also excluded: complicating malignant tumours; occupational, medicationrelated, genetic, or environmental disease aetiology; familial history of IPF; history of pulmonary hypertension, congenital heart disease, pulmonary vein occlusion, chronic obstructive pulmonary disease, or left-sided heart failure; and haemodialysis. Finally, pregnant, potentially pregnant, and lactating women were excluded.

\section{Collection of clinical information and serum samples}

The following subject data were recorded: sex, age, clinical manifestations, medical history, smoking history, laboratory examination results [lactate dehydrogenase ( $\mathrm{LDH})$, arterial blood gas analysis, sputum smear, sputum culture], HRCT findings, and PFT results [forced vital capacity (FVC), forced expiratory volume in 1 second (FEV1), FEV1/FVC ratio, total lung capacity (TLC), carbon monoxide diffusing capacity (DLCO)].

\section{KL-6 assay}

Blood samples were collected from eligible, fasted patients into coagulation-promoting vacuum tubes and centrifuged at 3,000 rpm for 10 minutes within 2 hours after collection to isolate serum for further analyses. Serum KL-6 levels were evaluated using the Lumipulse ${ }^{\circledR}$ G KL-6 (Krebs von den Lungen) kit (storage at $2-10{ }^{\circ} \mathrm{C}$; Sekisui Medical Co., Ltd., Tokyo, Japan) according to the manufacturer's instructions. The results were measured using an automated immunoassay system (LUMIPULSE G1200; Fujirebio, Inc., Tokyo, Japan).

\section{Patient evaluation}

IIP complicated by pulmonary infection was defined as the presence of coughing, expectoration, and fever, as well as an elevated peripheral white blood cell count, the onset or progression of patchy shadows on HRCT, and a positive sputum smear and/or sputum bacterial culture. An improved response to therapy was defined as follows: (I) no change or a reduction in ground-glass opacity on HRCT and (II) an increase of $>10 \%$ in the FVC and/or $>15 \%$ in the DLCO (actual value/predicted value, \%) and/ or an arterial blood gas analysis indicating improvement or normalisation [arterial oxygen partial pressure increase $\left(\mathrm{PaO}_{2}\right)$ of $>4 \mathrm{mmHg}$. CT results were interpreted by two independent imaging physicians. IIP progression was defined as an exacerbation of dyspnoea and fulfilment of the following criteria: (I) onset or progression of a ground-glass opacity on HRCT and (II) a decrease of $>10 \%$ in the FVC and/or $>15 \%$ in the DLCO (actual value/predicted value, \%) and/or an arterial blood gas analysis indicating progressive deterioration $\left(\mathrm{PaO}_{2}\right.$ of $\left.>4 \mathrm{mmHg}\right)$. A stable (and presumed favourable) response to therapy was defined by two or more of the following: (I) $10 \%$ change in TLC or VC, (II) $15 \%$ change in DLCO (actual value/predicted value, \%) and (III) no change in $\mathrm{PaO}_{2}$ (4 mmHg increase) achieved during a formal cardiopulmonary exercise test.

\section{Statistical analysis}

A database of all research data, including relevant clinical information (e.g., basic information, serum KL-6 concentrations, and CT imaging findings), was established. Statistical analyses were conducted using SPSS 22.0 (SPSS, Inc., Chicago, IL, USA) and Prism5.0 statistical software (GraphPad, Inc., La Jolla, CA, USA).

Differential analyses of the serum KL-6 concentrations and other basic parameters were conducted among different groups. Parametric data were analysed using a $t$-test, and the results are presented as means \pm standard deviations. Nonparametric data were analysed using the Wilcoxon rank sum test, and the results are presented as medians (interquartile ranges). A P value $<0.05$ indicates a statistically significant difference.

A receiver operating characteristic (ROC) curve was plotted using diagnosis test data, and the area under the ROC curve was determined to identify the optimal serum KL-6 concentration cut-off point for the diagnosis of IIP. The ROC curve data were also used to determine the diagnostic sensitivity, specificity, positive predictive value (PPV), odds ratio (OR), negative predictive value (NPV), misdiagnosis rate, missed rate, and accuracy. 
Table 1 Basic information about the study subjects in the two groups

\begin{tabular}{lccc}
\hline Group & IIP group & Non-IIP group & P value \\
\hline Cases, $\mathrm{n}(\%)$ & $75(71.43)$ & $30(28.57)$ & - \\
Sex (male/female, $\mathrm{n})$ & $46 / 29$ & $56.5(42.0-61.5)$ & 0.513 \\
Age (year) & $63.0(50.0-67.0)$ & $21.56 \pm 3.90[16]$ & 0.018 \\
BMI [n] & $24.54 \pm 2.98[57]$ & $7 / 18$ & 0.002 \\
Smoking history (yes/no) & $36 / 38$ & $86.20 \pm 18.09[16]$ & 0.567 \\
FVC\% [n] & $73.38 \pm 17.19[53]$ & $86.33(71.58-93)[16]$ & 0.006 \\
FEV1\% [n] & $79(63.48-88)[53]$ & $94.36 \pm 13.63[11]$ & 0.235 \\
TLC\% [n] & $71.54 \pm 15.76[52]$ & $104(99.5-108)$ & $<0.001$ \\
PaO 2 (mmHg) & $103.1(99.8-107.7)$ & $210.0(155.6-304.1)$ & 0.941 \\
LDH (U/mL) & $207.4(173.3-256.9)$ & $226(173.5-346.5)$ & 0.380 \\
KL-6 level (U/mL) & $1,096(565.0-1,544)$ & $<0.001$ &
\end{tabular}

Parametric data are presented as means \pm standard deviations; non-parametric data are presented as medians (interquartile ranges). KL-6, Krebs von den Lungen-6 antigen; IIP, idiopathic interstitial pneumonia; BMI, body mass index; FVC\%, forced vital capacity; FEV1\%, forced expiratory volume in 1 second; TLC\%, total lung capacity; $\mathrm{PaO}_{2}$, arterial partial pressure of oxygen; $\mathrm{LDH}$, lactate dehydrogenase.

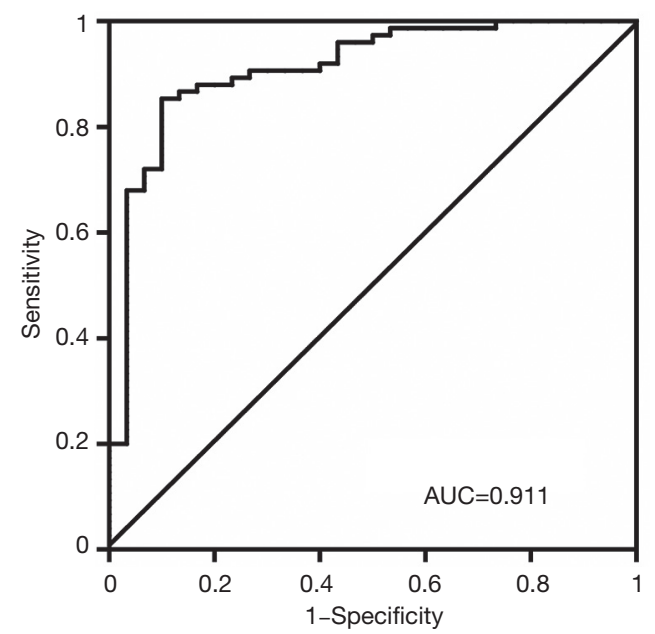

Figure 1 ROC curve analysis of the serum KL-6 level as a diagnostic marker of IIP. AUC, area under the curve; KL-6, Krebs von den Lungen-6 antigen; ROC, receiver operating characteristic; IIP, idiopathic interstitial pneumonia.

\section{Results}

\section{Basic information about the study subjects}

Interstitial pneumonia was found to be more common among elderly people (i.e., 60-70 years of age) and as expected, the mean age was significantly higher in the IIP group than in the non-IIP group $(\mathrm{Z}=-2.357, \mathrm{P}=0.018)$. Compared to the non-IIP group, the IIP group also had a significantly higher body mass index (BMI) $(\mathrm{t}=3.297$, $\mathrm{P}=0.002$ ) but significantly lower $\mathrm{FVC} \%$ and $\mathrm{TLC} \%$ values (FVC\%: $\mathrm{t}=2.583, \mathrm{P}=0.006$; TLC $\%: \mathrm{t}=4.456, \mathrm{P}<0.001$ ). Furthermore, the IIP group had a significantly higher serum KL-6 level, compared with the non-IIP group $(\mathrm{Z}=-6.561$, $\mathrm{P}<0.001)$. The groups did not differ significantly in terms of the $\mathrm{FEV} 1 \%, \mathrm{PaO}_{2}$, and $\mathrm{LDH}$ values (Table 1).

\section{ROC curve analysis of KL-6 as a diagnostic biomarker of IIP}

The ROC curve of the KL-6 diagnostic test is shown in Figure 1. The area under the ROC curve was 0.911 [95\% confidence interval (CI): 0.847-0.975, P<0.001). The Youden index was calculated based on the sensitivity and specificity of each diagnostic cut-off point in the ROC curve, and the point with the highest Youden index was selected as the optimal KL-6 cut-off point for the diagnosis of IIPs. The optimal cut-off value of $485 \mathrm{U} / \mathrm{mL}$ corresponded to a sensitivity of $85.33 \%$ (95\% CI: $75.27-92.44 \%$ ) and specificity of $90.00 \%$ (95\% CI: $73.47-$ $97.89 \%)$, indicating the relatively high diagnostic value of the KL-6 assay.

The optimal KL-6 cut-off point obtained from the Youden index for the IIP diagnosis was compared with 
Table 2 KL-6 levels and other parameters of patients with and without complicating infections

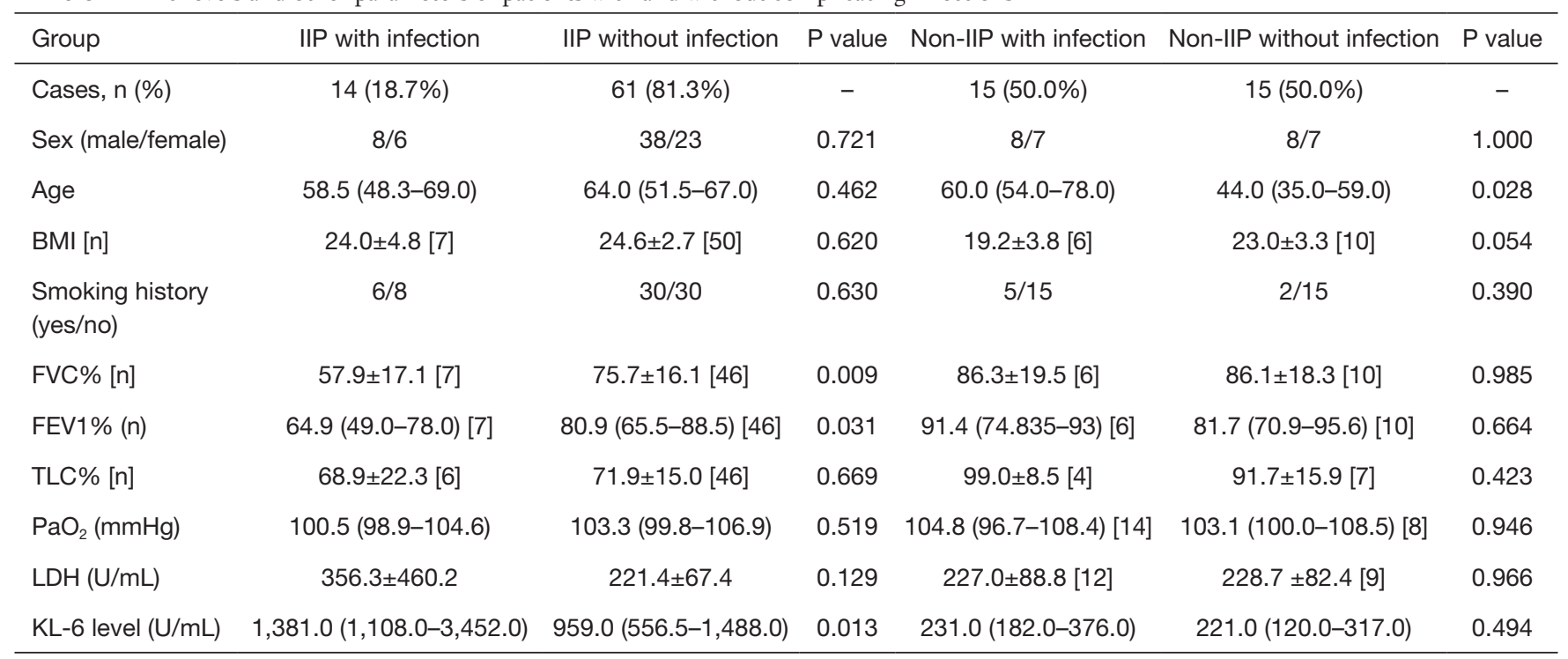

Parametric data are presented as means \pm standard deviations; non-parametric data are presented as medians (interquartile ranges). KL-6, Krebs von den Lungen-6 antigen; IIP, idiopathic interstitial pneumonia; BMI, body mass index; FVC\%, forced vital capacity; FEV1\%, forced expiratory volume in 1 second; TLC\%, total lung capacity; $\mathrm{PaO} 2$, arterial partial pressure of oxygen; LDH, lactate dehydrogenase.

the results of standard diagnostic methods, namely clinical manifestation, pathologic biopsy, and HRCT. In a comparison of the KL-6 diagnostic test with a clinical diagnosis of IIP, the use of KL-6 yielded 64 true positives, 3 false positives, 27 true negatives, and 11 false negatives with a false positive rate of $4 \%(3 / 75)$ and a false negative rate of $36.7 \%(11 / 30)$. A KL-6 level of $486 \mathrm{U} / \mathrm{mL}$ also yielded an accuracy of $86.67 \%$ (91/105), misdiagnosis rate of $10.00 \%$ (3/30), miss rate of $14.67 \%$ (11/75), PPV of $95.52 \%(64 / 67)$, NPV of $71.05 \%$ (27/38), and OR of 6.36. In other words, KL-6 could be used to diagnose IIP with a relatively high accuracy and predictive value. Furthermore, a consistency test yielded a Kappa value of 0.70 , indicating that the KL-6 diagnostic results were highly consistent with the clinical diagnostic results.

\section{KL-6 levels in IIP patients with complicating pulmonary infections}

Among the 75 IIP patients, 14 presented with pulmonary infections. A comparison between these patients and those without pulmonary infections $(n=61)$ revealed that the former had significantly higher KL-6 levels $(Z=-2.475$, $\mathrm{P}=0.013)$. Regarding lung function, patients with pulmonary infections had a significantly lower FVC\% and FEV1\% relative to those without pulmonary infections (FVC\%: $\mathrm{t}=4.108, \mathrm{P}=0.009$; FEV1\%: $\mathrm{Z}=-1.866, \mathrm{P}=0.031$ ), whereas no significant difference in TLC\% was observed $(t=0.430$, $\mathrm{P}=0.669)$. However, there was no significant relationship between the level of KL-6 in non-IIP patients $(n=30)$ with or without pulmonary infection (Table 2).

\section{Relationship between disease progression and dynamic changes in KL-6 levels}

We followed 19 patients diagnosed with IIPs who underwent CT imaging and KL-6 testing during return visits within a 2 -year period. A combination of the IIP progression assessment criteria, CT imaging findings, and clinical signs revealed that among these 19 returnvisit patients, 7 had deteriorated, 7 had improved, and 5 remained stable. For these patients, treatments were mainly administered as prophylaxis against fibrosis and infection, for cough with phlegm, and for hormone therapy. The treatments administered to these 19 followed patients are listed in the Table S1.

Our dynamic follow-up of the KL-6 levels from pretreatment to post-treatment allowed us to evaluate IIP progression among the participating patients. As shown in Figure 2, the dynamic changes in KL-6 concentrations over 


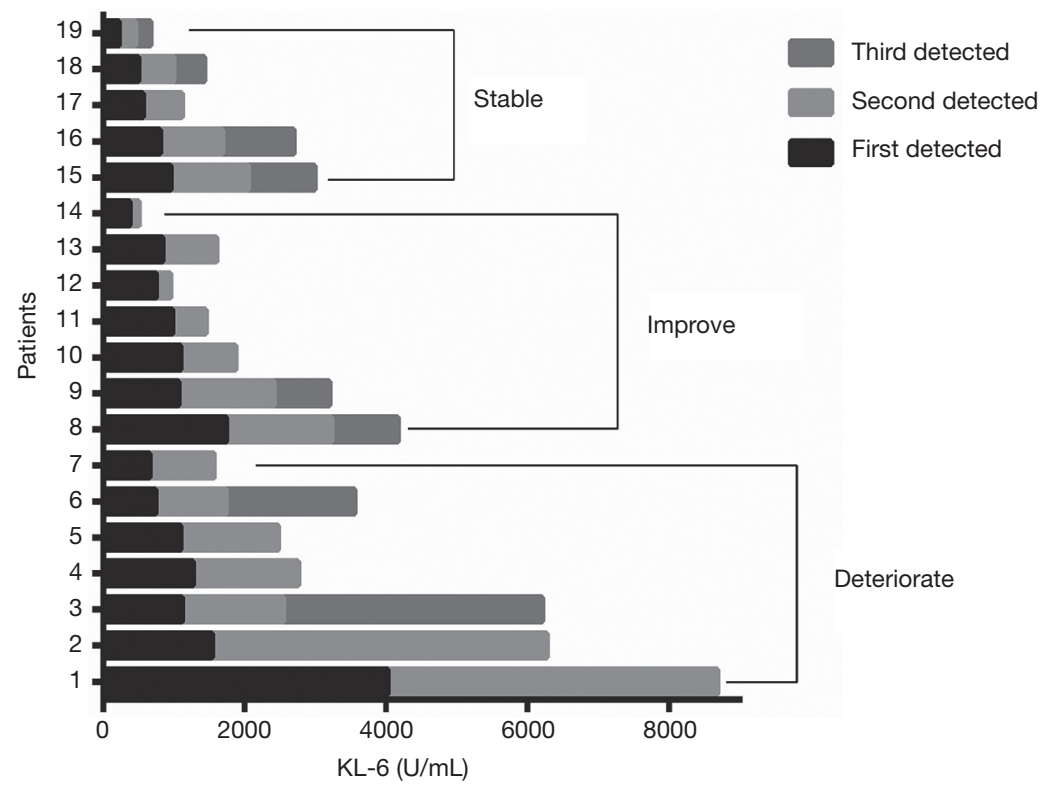

Figure 2 Analysis of the relationship between the dynamic changes in KL-6 levels and disease progression in patients with idiopathic interstitial pneumonia who were followed up in a range of 2 years. KL-6, Krebs von den Lungen-6 antigen.
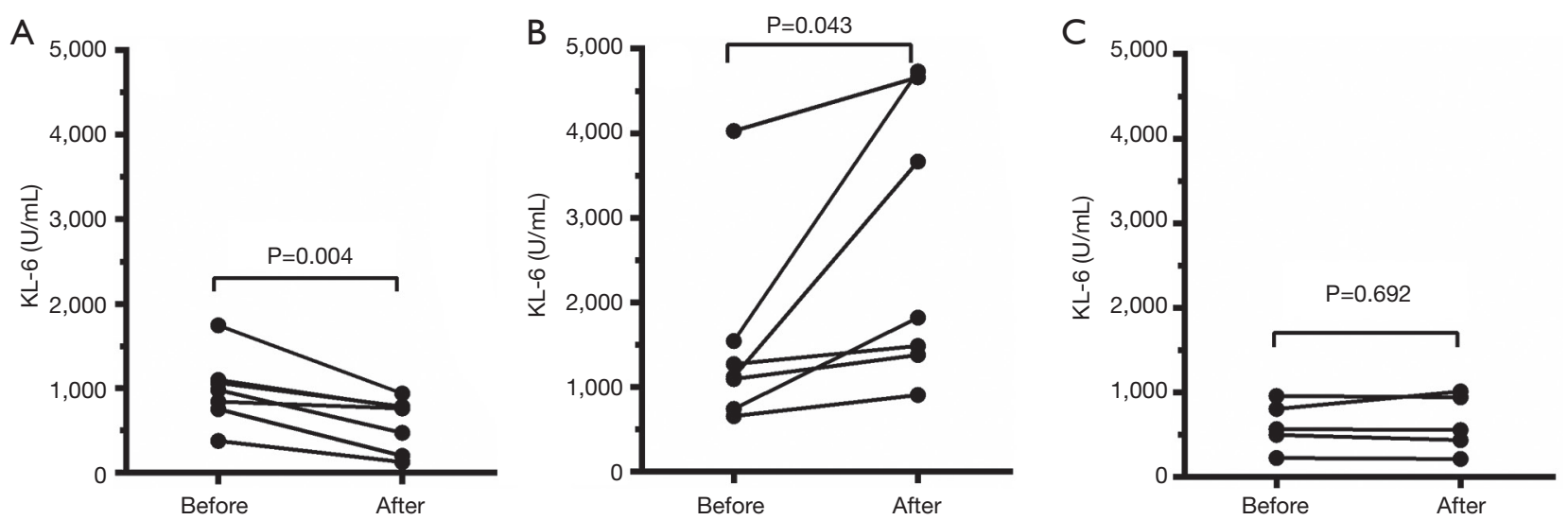

Figure 3 Comparison of the KL-6 levels in patients with idiopathic interstitial pneumonia from before to after treatment. Statistical differences were determined using the Wilcoxon matched-pairs signed-rank test. A P value $<0.05$ indicates a statistically significant difference between time points. Panels A, B, and C depict the mean KL-6 levels among cases that exhibited improvement, deterioration, and stable disease, respectively. KL-6, Krebs von den Lungen-6 antigen.

multiple time points intuitively reflected disease progression and could be used to predict the patients' clinical outcomes. The 7 deteriorated cases exhibited significantly higher posttreatment KL-6 levels relative to the pre-treatment levels $(\mathrm{P}=0.043)$; by contrast, the 7 improved cases exhibited significantly lower post-treatment KL-6 levels relative to the pre-treatment levels $(\mathrm{P}=0.004)$, with a significant reduction in the post-treatment positive rate. No significant changes were observed for the 5 stable cases $(\mathrm{P}=0.692)$ (Figure 3).

\section{Discussion}

IIPs are heterogeneous, diffuse ILDs with unknown 
aetiologies. These diseases affect not only the lung interstitium, but also the lung parenchyma, alveolar cavity, alveolar epithelium, peripheral airways, and capillaries. Although HRCT examination, PFTs, blood gas analysis, and LDH detection are commonly used to detect IIPs, these insensitive modalities operate within a narrow relative range and usually provide non-specific diagnostic information. Additionally, IIPs are often complicated by bacterial infections or inflammation in other organs.

Many recent studies have noted that an increased KL-6 level is a positive and clinically significant indicator for the diagnosis and differential diagnosis of IIP (13). In China, studies of KL-6 have mainly focused on significant increases in the serum levels of this antigen in the context of different types of ILDs, including IPF, CVD-IP, and HP, and the results have suggested the potential diagnostic use of this parameter. In other countries, researchers have reported distinctly elevated serum KL-6 levels in patients diagnosed with IIP, radiation pneumonitis, pulmonary alveolar proteinosis, CTD-related interstitial pneumonia, non-small cell lung cancer, bronchopulmonary dysplasia in preterm infants, and neonatal high-permeability pulmonary oedema (14-17). Our observation of significantly higher serum KL-6 levels in patients with IIP relative to those in patients with other lung diseases agreed with the findings of Kohno and $\operatorname{Vij}(11,18)$.

In our study, we used a ROC curve analysis and area under the curve of 0.911 to determine a serum KL-6 cut-off value of $485 \mathrm{U} / \mathrm{mL}$. This cut-off value yielded a sensitivity of $85.33 \%$ and specificity of $90.00 \%$, indicating a relatively good diagnostic value. Previously, Kobayashi et al. analysed 118 patients with IIP and determined a relatively higher KL-6 cut-off range of 550-700 U/mL; in that study, however, the ROC curve analysis of the discriminatory ability of KL-6 yielded a cut-off value of $476 \mathrm{U} / \mathrm{mL}$ for distinguishing IPF patients ( $\mathrm{n}=65)$ from control subjects (19). As the KL-6 cut-off values for diagnosis differ among the different types of ILDs, a cut-off value of $500 \mathrm{U} / \mathrm{mL}$ is commonly used as the clinical diagnostic standard.

Previously, Ishii et al. diagnosed ILDs based on the accuracy, sensitivity, specificity, and likelihood ratio (LR) and proposed the use of alveolar KL-6 and mucin 1 (MUC1) expression to identify most cases of ILDs among patients who had been diagnosed with other benign lung diseases, such as pneumonia (20). Using clinical manifestations, pathologic biopsy findings, and HRCR results as diagnostic standards, we calculated the accuracy, PPV and OR of the KL-6 test for IIP diagnosis with a serum KL-6 level of
$485 \mathrm{U} / \mathrm{mL}$ as the cut-off point. The results suggest that the serum KL-6 level exhibited a relatively high accuracy and predictive value for the diagnosis of IIPs. Furthermore, the Kappa value of 0.70 from a consistency test indicated good agreement between the serum KL-6-based and clinical IIP diagnostic results.

Previous studies reported that the serum KL-6 levels did not change significantly among patients who were additionally diagnosed with bacterial or fungal pulmonary alveolitis $(21,22)$. In the present study, we observed significantly higher KL-6 levels and significantly lower $\mathrm{FVC} \%$ and $\mathrm{FEV} \%$ values in IIP patients with complicating pulmonary infections, compared to those without such infections. Infection is the main cause of an AE-IPF, for which the latest guidelines do not distinguish infection from acute exacerbation. Several studies found that the serum levels of KL-6 correlated with the extent and activity of IPF $(23,24)$. Therefore, the presence of infection should be considered in patients who present with abnormally high KL-6 concentrations during clinical testing.

In IIP patients, the serum KL-6 level has previously been associated with therapeutic efficacy and disease progression (9). In the present study, a follow-up analysis of IIP patients revealed that those clinically diagnosed with post-treatment deterioration had significantly elevated KL-6 levels, whereas those who improved with treatment exhibited dramatic decreases in both KL-6 levels and the KL-6-positive rate. By contrast, patients with stable conditions did not exhibit apparent changes in KL-6 levels. Therefore, a dynamic change in the KL-6 level appears to reflect IIP progression. Our follow-up analysis also suggested that the KL-6 level could be used to estimate the clinical outcomes of IIP patients. Similarly, a previous study found that the KL-6 level could be used to evaluate the activities and predict the prognoses of different types of ILDs (25). Among ILD patients, those whose KL-6 levels decreased significantly during therapy had good responses to therapy (26), whereas an increased KL-6 level was associated with a low survival rate (19). Oguz et al. reported that the KL-6 level could be used as a predictive factor to identify the clinical development of ILD before it can be detected via imaging (27). The KL-6-based test has a relatively high diagnostic value, sensitivity and specificity for IIP, and this simple, rapid, and highly repeatable process places minimal physiological burdens on patients. Furthermore, the quantitative results of KL-6 tests can be observed and managed via digital means and are thus conducive to disease monitoring over time. Therefore, the 
serum KL-6 level may become a reliable reference indicator in the clinical diagnosis of IIP.

This study had some limitations of note. First, the low return visit rate among IIP patients affected the followup study. Second, IIPs are more prevalent among middleaged and elderly populations, which led to a significant age difference between the experimental and control group in our study. The recruitment of more closely age-matched subjects would have increased the clinical reliability of our findings. Regardless of these limitations, our study represents a preliminary exploration of the clinical diagnostic and prognostic value of blood KL-6 levels in patients with IIP and other lung diseases. Our findings suggest that the presence of complications or infections should be considered in an IIP patient who exhibits an abnormal increase in KL-6 levels.

In conclusions, the serum KL-6 level was found to be highly valuable for the diagnosis of IIP. Specifically, this marker could be used to distinguish IIP from non-IIP after setting a cut-off point of $485 \mathrm{U} / \mathrm{mL}$. Moreover, IIP patients with pulmonary infection had significantly higher serum levels of KL-6, compared to those without pulmonary infection. Our findings suggest that the current or previous infection status should be investigated in patients with very high serum concentrations of KL-6. Our follow-up analysis further indicated that the KL-6 level could be used as an index of IIP progression and a predictor of clinical outcomes.

\section{Acknowledgements}

Funding: This study was funded by the National Natural Science Foundation of China (Project No.: 81572063, 81601394), Medicine and Health Care Technology Projects of Guangzhou (Project No.: 2017A013010017), Open Project of State Key Laboratory of Respiratory Disease (SKLRD2016OP003). The funders had no role in study design, data collection and analysis, decision to publish, or preparation of the manuscript.

\section{Footnote}

Conflicts of Interest: The authors have no conflicts of interest to declare.

Ethical Statement: This study was approved by the Medical Ethics Committee of the First Affiliated Hospital of Guangzhou Medical University (ethics approval no. gyfyy-2016-73). All experiments were performed in accordance with the relevant guidelines and regulations of the Ethics Committee of First Affiliated Hospital of Guangzhou Medical University.

\section{References}

1. Travis WD, Costabel U, Hansell DM, et al. An official American Thoracic Society/European Respiratory Society statement: Update of the international multidisciplinary classification of the idiopathic interstitial pneumonias. Am J Respir Crit Care Med 2013;188:733-48.

2. Coultas DB, Zumwalt RE, Black WC, et al. The epidemiology of interstitial lung diseases. Am J Respir Crit Care Med 1994;150:967-72.

3. Raghu G, Weycker D, Edelsberg J, et al. Incidence and prevalence of idiopathic pulmonary fibrosis. Am J Respir Crit Care Med 2006;174:810-6.

4. Nathan SD, Shlobin OA, Weir N, et al. Long-term course and prognosis of idiopathic pulmonary fibrosis in the new millennium. Chest 2011;140:221-9.

5. Juarez MM, Chan AL, Norris AG, et al. Acute exacerbation of idiopathic pulmonary fibrosis-a review of current and novel pharmacotherapies. J Thorac Dis 2015;7:499-519.

6. American Thoracic Society. Idiopathic pulmonary fibrosis: diagnosis and treatment. International consensus statement. American Thoracic Society (ATS), and the European Respiratory Society (ERS). Am J Respir Crit Care Med 2000;161:646-64.

7. Kohno N, Inoue $\mathrm{Y}$, Hamada $\mathrm{H}$, et al. Difference in serodiagnostic values among KL-6-associated mucins classified as cluster 9. Int J Cancer Suppl 1994;8:81-3.

8. Hirasawa Y, Kohno N, Yokoyama A, et al. KL-6, a human MUC1 mucin, is chemotactic for human fibroblasts. Am J Respir Cell Mol Biol 1997;17:501-7.

9. Ishikawa N, Hattori N, Yokoyama A, et al. Utility of KL-6/MUC1 in the clinical management of interstitial lung diseases. Respir Investig 2012;50:3-13.

10. Kohno N. Serum marker KL-6/MUC1 for the diagnosis and management of interstitial pneumonitis. J Med Invest 1999;46:151-8.

11. Kohno N, Kyoizumi S, Awaya Y, et al. New serum indicator of interstitial pneumonitis activity. Sialylated carbohydrate antigen KL-6. Chest 1989;96:68-73.

12. Zhu C, Zhao YB, Kong LF, et al. The expression and clinical role of KL-6 in serum and BALF of patients with different diffuse interstitial lung diseases. Zhonghua Jie He 
He Hu Xi Za Zhi 2016;39:93-7.

13. Ohnishi H, Yokoyama A, Kondo K, et al. Comparative study of KL-6, surfactant protein-A, surfactant protein-D, and monocyte chemoattractant protein-1 as serum markers for interstitial lung diseases. Am J Respir Crit Care Med 2002;165:378-81.

14. Okamoto T, Fujii M, Furusawa H, et al. The usefulness of KL-6 and SP-D for the diagnosis and management of chronic hypersensitivity pneumonitis. Respir Med 2015;109:1576-81.

15. Masuda T, Hirano C, Horimasu Y, et al. The extent of ground-glass attenuation is a risk factor of chemotherapyrelated exacerbation of interstitial lung disease in patients with non-small cell lung cancer. Cancer Chemother Pharmacol 2018;81:131-9.

16. Dilli D, Ozyazici A, Dursun A, et al. Predictive values of plasma KL-6 in bronchopulmonary dysplasia in preterm infants. Turk J Med Sci 2017;47:621-6.

17. Nakamura T, Nakamura M, Takahashi N. Neonatal highpermeability pulmonary edema based on serial cytokine profiles and KL-6 in serum: case report. Fukushima J Med Sci 2017;63:22-7.

18. Vij R, Noth I. Peripheral blood biomarkers in idiopathic pulmonary fibrosis. Transl Res 2012;159:218-27.

19. Hamai K, Iwamoto H, Ishikawa N, et al. Comparative Study of Circulating MMP-7, CCL18, KL-6, SP-A, and SP-D as Disease Markers of Idiopathic Pulmonary Fibrosis. Dis Markers 2016;2016:4759040.

Cite this article as: Zheng P, Liu X, Huang H, Guo Z, Wu G, Hu H, Cai C, Luo W, Wei N, Han Q, Sun B. Diagnostic value of KL-6 in idiopathic interstitial pneumonia. J Thorac Dis 2018;10(8):4724-4732. doi: 10.21037/jtd.2018.07.54
20. Ishii H, Mukae H, Kadota J, et al. High serum concentrations of surfactant protein A in usual interstitial pneumonia compared with non-specific interstitial pneumonia. Thorax 2003;58:52-7.

21. Nakajima M, Manabe T, Mitekura H, et al. Levels of serum KL-6 in a patient with drug-induced pneumonitis. Nihon Kyobu Shikkan Gakkai Zasshi 1997;35:813-7.

22. Nakajima M, Manabe T, Niki Y, et al. Serum KL-6 level as a monitoring marker in a patient with pulmonary alveolar proteinosis. Thorax 1998;53:809-11.

23. Sakamoto K, Taniguchi H, Kondoh Y, et al. Serum KL-6 in fibrotic NSIP: Correlations with physiologic and radiologic parameters. Respir Med 2010;104:127-33.

24. Ichiyasu H, Ichikado K, Yamashita A, et al. Pneumocyte biomarkers KL-6 and surfactant protein D reflect the distinct findings of high-resolution computed tomography in nonspecific interstitial pneumonia. Respiration 2012;83:190-7.

25. Yokoyama A, Kondo K, Nakajima M, et al. Prognostic value of circulating KL-6 in idiopathic pulmonary fibrosis. Respirology 2006;11:164-8.

26. Ohshimo S, Ishikawa N, Horimasu Y, et al. Baseline KL-6 predicts increased risk for acute exacerbation of idiopathic pulmonary fibrosis. Respir Med 2014;108:1031-9.

27. Oguz EO, Kucuksahin O, Turgay M, et al. Association of serum KL-6 levels with interstitial lung disease in patients with connective tissue disease: a cross-sectional study. Clin Rheumatol 2016;35:663-6. 


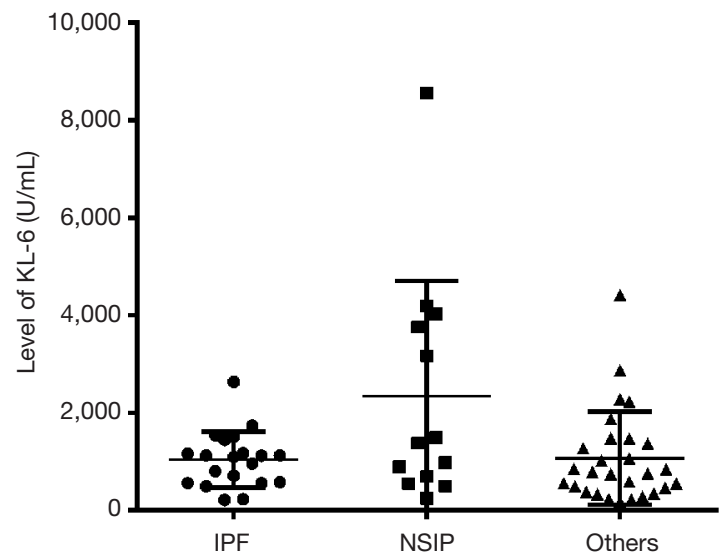

Figure S1 Comparative analysis of KL-6 levels among different types of idiopathic interstitial pneumonia. The y-axis indicates the serum KL-6 level (U/mL), while the $\mathrm{x}$-axis indicates different types of idiopathic interstitial pneumonia. Data are presented as means \pm standard deviations; Statistical differences were determined using the Wilcoxon matched-pairs signed-rank test. No statistically significant difference was observed among three groups. KL-6, Krebs von den Lungen-6 antigen; IPF, idiopathic pulmonary fibrosis; UIP, usual interstitial pneumonia; Others, RB-ILD, DIP, COP, AIP, etc. RB-ILD, respiratory bronchiolitis-interstitial lung disease; DIP, desquamative interstitial pneumonia; COP, cryptogenic organising pneumonia; AIP, acute interstitial pneumonia.

Table S1 Treatments administered to 19 patients with IIP who were followed

\begin{tabular}{|c|c|c|}
\hline Group & Patients & Treatment \\
\hline \multirow[t]{7}{*}{ Improvement } & 1 & Pirfenidone, acetylcysteine, anti- infectives \\
\hline & 2 & Corticosteroid, anti-infectives, stomach proctants, anti-osteoporosis, stomach protection, et al. \\
\hline & 3 & Corticosteroid, anti-infectives, anti-osteoporosis, stomach protection, antilipemic, antidiabetic, anti-allergic, et al. \\
\hline & 4 & Acetylcysteine \\
\hline & 5 & Acetylcysteine, corticosteroid, anti-infectives, anti-osteoporosis, stomach protection, antidiabetic, hypotensor, et al. \\
\hline & 6 & Acetylcysteine, corticosteroid, anti-osteoporosis, stomach protection, liver protection, et al. \\
\hline & 7 & Corticosteroid, anti-osteoporosis, stomach protection \\
\hline \multirow[t]{7}{*}{ Deterioration } & 8 & $\begin{array}{l}\text { Acetylcysteine, corticosteroid, immunosuppressant, anti-infectives, stomach protection, liver protection, } \\
\text { anti-osteoporosis, et al. }\end{array}$ \\
\hline & 9 & Acetylcysteine, corticosteroid, anti-infectives, stomach protection, antiallergic, antidiabetic, antilipemic, et al. \\
\hline & 10 & Acetylcysteine, corticosteroid, anti-infectives, anti-osteoporosis, stomach protection, hypotensor, et al. \\
\hline & 11 & Acetylcysteine, corticosteroid, anti-infectives, anti-osteoporosis, stomach protection \\
\hline & 12 & Pirfenidone, acetylcysteine, corticosteroid, anticoagulant, stomach protection, antilipemic, antidiabetic, et al. \\
\hline & 13 & $\begin{array}{l}\text { Acetylcysteine, corticosteroid, immunosuppressant, anti-infectives, anti-osteoporosis, stomach protection, } \\
\text { hypotensor }\end{array}$ \\
\hline & 14 & Acetylcysteine, corticosteroid, anti-osteoporosis, liver protection, stomach protection, analgesic, et al. \\
\hline \multirow[t]{5}{*}{ Stable } & 15 & Acetylcysteine, corticosteroid, anti-infectives, anti-osteoporosis, stomach protection, et al. \\
\hline & 16 & Acetylcysteine, corticosteroid, anti-infectives, anti-osteoporosis, stomach protection, antidiabetic, et al. \\
\hline & 17 & Pirfenidone, acetylcysteine, anti-infectives, anti-osteoporosis, stomach protection, et al. \\
\hline & 18 & Acetylcysteine, anti-infectives, anti-osteoporosis, stomach protection, antidiabetic \\
\hline & 19 & Pirfenidone, acetylcysteine \\
\hline
\end{tabular}

Corticosteroid: methylprednisolone, prednisone, etc.; anti-infectives: antibiotic, antifungal, and antiviral agents; anti-osteoporosis: calcitriol; stomach protection: omeprazole, lansoprazole, domperidone, hydrotalcite, etc.; liver protection: polyene phosphatidylcholine capsules, diammonium glycyrrhizinate, silibin meglumine; analgesic: codeine phosphate tablets, celecoxib; antilipemic: simvastatin, fenofibrate, etc.; antidiabetic: insulin, nateglinide, etc.; hypotensor: bisoprolol, amlodipine, felodipine. In addition to the above treatments, some patients may have also used drugs for other comorbidities such as asthma, coronary heart disease, and rheumatoid arthritis. IIP, idiopathic interstitial pneumonia. 